\title{
Red Blood Cell Distribution Width as a Predictive Marker for Coronary Artery Lesions in Patients with Kawasaki Disease
}

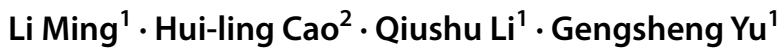

Received: 21 December 2020 / Accepted: 3 May 2021 / Published online: 25 May 2021

(c) The Author(s) 2021, corrected publication 2021

\begin{abstract}
This study aimed to investigate the association between red blood cell distribution width (RDW) and the risk of coronary artery lesions (CALs) in patients with Kawasaki disease (KD). A total of 1355 patients who met the diagnostic criteria for KD were reviewed between January 2018 and December 2019, including 636 patients with CALs and 719 patients without CALs. Blood samples for RDW were obtained at admission (before intravenous immunoglobulin treatment). A logistic regression analysis was performed, and a receiver operating characteristic curve was constructed to determine the prognostic value of RDW standard deviation (RDW-SD) and RDW coefficient of variation (RDW-CV). The study was registered at www.chictr.org.cn, No.: ChiCTR 2000040980. The results showed that RDW-SD increased in patients with complete KD and CALs compared with patients with complete KD without CALs (39 fL vs. $38 \mathrm{fL}$, respectively; $p=0.000$ ). RDW-CV in patients with complete KD and CALs was significantly higher compared with patients with completed KD without CALs $(p=0.000)$. Further multivariate logistic regression analysis revealed that RDW-SD was an independent marker of CALs in patients with complete $\mathrm{KD}(p=0.001)$, but no association was found between RDW-CV and CALs. The area under the curve of RDW-SD for predicting CALs in patients with complete KD was 0.606 (95\% confidence interval 0.572-0.640; $p=0.000$ ) with a sensitivity and specificity of $61 \%$ and $55 \%$, respectively, when the optimal cut-off value of RDW-SD was $38.5 \mathrm{fL}$. RDW-CV increased in patients with incomplete KD and CALs compared with patients without CALs (13.55\% vs $13.3 \%$, respectively; $p=0.004$ ), and multivariate logistic regression analysis revealed that RDW-CV was an independent marker of CALs in patients with incomplete $\mathrm{KD}(p=0.021)$. The area under the curve of RDW-CV for predicting CALs in patients with incomplete KD was 0.597 (95\% confidence interval $0.532-0.661 ; p=0.004)$ with a sensitivity and specificity of $40 \%$ and $77 \%$, respectively, when the optimal cut-off value of RDW-SD was $13.85 \%$. Conclusion: RDW can be used as an independent predictive marker of CALs in patients with KD, but the type of KD should be considered. RDW-SD was an independent marker of CALs in patients with complete KD, while RDW-CV was a predictor of incomplete KD.
\end{abstract}

Keywords Kawasaki disease $\cdot$ Coronary artery lesion $\cdot$ Red blood cell distribution

Gengsheng Yu

yugengsheng2020@163.com

Li Ming

710315177@qq.com

Hui-ling Cao

C16623000406@163.com

Qiushu Li

370391137@qq.com

1 Chongqing Key Laboratory of Pediatrics, China International Science and Technology Cooperation Base of Child Development and Critical Disorders, Department of Heart Centre, National Clinical Research Center for Child Health and Disorders, Ministry of Education Key Laboratory of Child Development and Disorders, Children's Hospital of Chongqing Medical University, Chongqing 400014, China
2 Chongqing Key Laboratory of Pediatrics, China International Science and Technology Cooperation Base of Child Development and Critical Disorders, Department of Neonatology, National Clinical Research Center for Child Health and Disorders, Ministry of Education Key Laboratory of Child Development and Disorders, Children's Hospital of Chongqing Medical University, Chongqing, China 


$\begin{array}{ll}\text { Abbreviations } \\ \text { CALs } & \text { Coronary artery lesions } \\ \text { KD } & \text { Kawasaki disease } \\ \text { RDW } & \begin{array}{l}\text { Red blood cell distribution width } \\ \text { RDW-SD }\end{array} \\ \begin{array}{l}\text { Red blood cell distribution width standard } \\ \text { deviation }\end{array} \\ \text { RDW-CV } & \begin{array}{l}\text { Red blood cell distribution width coefficient } \\ \text { of variation }\end{array}\end{array}$

\section{Introduction}

Kawasaki disease (KD), which is an acute systemic vasculitis of unknown cause, usually occurs in children $<5$ years of age [1]. The most serious complications of KD are coronary artery lesions (CALs), such as dilatation and aneurysm. The prevalence of CALs can be minimized if treatment with intravenous immunoglobulin (IVIG) is administered before the 10th day of disease [2]. However, approximately 10-15\% of patients with KD are IVIG resistant and are thus at an increased risk of developing CALs [3,4]. CALs may persist and progress to obstruction or stenosis, leading to ischemia and even sudden death. Therefore, KD is the leading cause of acquired heart disease in developed countries [5, 6]. The prognosis, follow up, and long-term management of patients with KD depend mainly on the severity of CALs. Clinicians tend to treat patients with KD who are at a high risk of CALs more aggressively to block the inflammatory response and minimize progression to CALs. Although risk factors for CALs have been studied extensively, no specific biological markers for predicting CALs in clinical practice have been established thus far.

Red cell distribution width (RDW), which is a marker of variation in the size of circulating erythrocytes (anisocytosis), is routinely measured in clinical practice as part of the complete blood count (CBC). Traditionally, RDW is used in the differential diagnosis of anemia [7]. In 2007, Felker et al. [8] first discovered that an increase in RDW is an independent predictor of morbidity and mortality in patients with chronic heart failure. Growing interest has been expressed in the clinical value of RDW. An increasing number of studies have demonstrated that a higher RDW is a prognostic factor for various cardiovascular system diseases, such as coronary artery disease [9], hypertension [10], heart failure [7, 8, 11], peripheral artery disease [12], coronary artery ectasia [13], and congenital heart disease [14]. However, studies on the relationship between RDW and CALs in patients with KD are limited, and these studies mainly focus on the RDW coefficient of variation (RDW-CV). RDW-CV is calculated by dividing the standard deviation (SD) of red blood cell (RBC) volume by mean erythrocyte volume and multiplying by $100 \%$ to express the result as a percentage [15]. Because information might be lost by expressing RDW relative to MCV, Hoffmann et al. [16] proposed that future studies on the prognostic value of RDW should focus on RDW-SD. Tonelli et al. [17] suggested that RDW-SD may be more useful as a prognostic marker than RDW-CV in the general population. Therefore, in the present study, we aimed to investigate the relationship between RDW, including RDW-CV and RDW-SD, and CALs in patients with KD.

\section{Materials and Methods}

\section{Patient Selection}

Between January 2018 and December 2019, this retrospective study was performed on 1355 patients with KD admitted to the Children's Hospital of Chongqing Medical University. The inclusion criteria were as follows: $(1) \leq 18$ years of age; (2) acute disease stage; (3) diagnosis of KD according to the criteria established by the American Heart Association. The exclusion criteria were (1) incomplete clinical data; (2) IVIG treatment at other medical institutions before admission/no IVIG treatment during hospitalization; (3) congenital heart disease, cardiomyopathy, myocarditis, valvular heart disease, severe arrhythmia, heart failure, or other heart disease; (4) moderate-to-severe anemia, leukemia, multiple myeloma, myelodysplastic syndrome, or other blood system disease.

The present study protocol was reviewed and approved by the Ethics Committee of the Children's Hospital Affiliated to Chongqing Medical University, and with the its approval, this study required no conformed consent. All methods were performed in accordance with Declaration of Helsinki and the relevant guidelines.

\section{Data Collection}

Demographic characteristics, laboratory data, and echocardiographic data were collected. Laboratory tests, including white blood cell counts, platelet counts, hemoglobin, mean erythrocyte volume, RDW-CV, RDW-SD, neutrophil percentage, lymphocyte percentage, eosinophil percentage, erythrocyte sedimentation rate, serum C-reactive protein, procalcitonin, glutamyltranspeptidase, alanine transaminase, aspartate aminotransferase, total bile acid, total bilirubin, albumin, uric acid, creatinine, serum sodium, fibrinogen, and activated partial thromboplastin time were measured in all subjects before IVIG treatment. RDW-CV and RDW-SD were determined using the XS-800i (Sysmex, Kobe, Japan) automated hematology analyzer. The normal range of RDW$\mathrm{CV}(\%)$ in our laboratory was less than $15.5 \%$, and the normal range of RDW-SD was between 40 and $80 \mathrm{fL}$.

Echocardiography was used to detect CALs during hospitalization. Due to lack of height data, no mean body surface area (BSA)-adjusted Z-score was available. CALs 
were defined according to an internal lumen diameter of $>2.5 \mathrm{~mm}$ in patients $<3$ years of age, $>3 \mathrm{~mm}$ in patients aged 3-9 years, and $>3.5 \mathrm{~mm}$ in patients aged $9-14$ years; an internal segment diameter $\geq 1.5$ times that of an adjacent segment; and an irregular lumen [18].

\section{Statistical Analysis}

Continuous variables are expressed as mean \pm standard deviation or median (25th-75th percentile) if non-normally distributed and were compared using independent-samples $t$ tests or Mann-Whitney $U$ tests between study groups. The normality of distribution of variables was checked using the Kolmogorov-Smirnov test. Categorical variables are expressed as percentages and were analyzed with the Chisquared test. To evaluate the independent effects of RDW on CALs in patients with $\mathrm{KD}$, a multivariate logistic regression analysis was performed. This analysis included age, sex and variables with a $p$ value of $<0.05$. The area under the receiver operating characteristic (ROC) curve (AUC) was analyzed to assess the predictive accuracy of RDW for CALs and to identify the optimal cut-off point. SPSS 25.0 (IBM Corporation, Armonk, New York, USA) was used for statistical analysis. A $p$ value $<0.05$ was considered statistically significant.

\section{Results}

A total of 1355 patients (856 males and 499 females) were enrolled in this study. The ages ranged from 2 to 184 months (median age: 28 months). Two hundred and ninety-five of those $(21.8 \%)$ had incomplete KD and six hundred and thirty-six developed CALs despite receiving IVIG.

\section{Characteristics of Patients with Complete KD and Incomplete KD}

We performed a subgroup analysis of the types of KD. In the subgroup of patients with complete $\mathrm{KD}$, compared with the group without CALs, RDW-CV, RDW-SD, glutamyltranspeptidase, procalcitonin, male sex and the frequency of IVIG resistant were significantly higher in patients with CALs (Table 1). On the contrary, hemoglobin, AST/ALT, albumin, and serum sodium were significantly lower in patients with CALs (Table 1). In the incomplete Kawasaki disease subgroup, male sex and RDW-CV levels in the CALs group were significantly higher than those in the without CALs (Table 2). While mean erythrocyte volume and total bilirubin were significantly lower in patients with CALs (Table 2).

\section{Independent Risks for Predicting CALs by Multivariate Analysis}

To evaluate the independent effects of RDW on CALs in patients with KD, we performed a multivariate logistic analysis. In patients with complete KD, a high RDW-SD was associated with CALs after adjusting for age, sex, hemoglobin, glutamyltranspeptidase, procalcitonin, AST/ALT, albumin, serum sodium, RDW-CV and IVIG resistance [odds ratio 1.115 (95\% confidence interval 1.046-1.188); $p=0.001]$ (Table 3). The multivariate logistic regression model also showed that age [odds ratio 1. 066 (95\% confidence interval 1.000-1.012); $p=0.050$ ], male sex [odds ratio 0.669 (95\% confidence interval 0.503-0.888); $p=0.006$ ], and hemoglobin(odds ratio 0.974 [95\% confidence interval $0.958-0.991] ; p=0.003$ ) were closely associated with CALs (Table 3). After adjusting for age, sex, mean erythrocyte volume and total bilirubin, the high RDW-CV levels was an independent risk factor for the development of CALs in patients with incomplete KD [odds ratio 1.428 (95\% confidence interval 1.056-1.930); $p=0.021$ ] (Table 3).

\section{ROC Analysis}

According to the ROC curve analysis, the optimal cut-off value of RDW-SD in patients with complete KD for predicting CALs was $38.5 \mathrm{fL}$, with a sensitivity of $61 \%$ and a specificity of $55 \%$ (AUC was $0.606,95 \%$ confidence interval $0.572-0.640 ; p=0.000 ;$ Fig. 1$)$. The optimal cut-off value of RDW-CV in patients with incomplete $\mathrm{KD}$ for predicting coronary artery lesions was 13.85 , with a sensitivity of $40 \%$ and a specificity of $77 \%$ (AUC was $0.597,95 \%$ confidence interval 0.532-0.661; $p=0.004$; Fig. 2).

\section{Discussion}

The present study demonstrated that a high RDW-SD is an independent marker of CALs in patients with complete KD. We found this association to be independent of multiple potential confounding factors, including age, sex, IVIG resistant, hemoglobin, RDW-CV, glutamyltranspeptidase, procalcitonin, AST/ALT, albumin, and serum sodium. Additionally, the AUC of RDW-SD for the predictor of CALs in patients with complete KD was 0.606 (95\% confidence interval $0.572-0.640 ; p=0.000$ ), with a sensitivity and a specificity value of $61 \%$ and $55 \%$, respectively, when the optimal cut-off value of RDW-SD was $38.5 \mathrm{fL}$. While in patients with incomplete KD, we found that a high RDW$\mathrm{CV}$ is an independent marker of CALs after adjusting for age, sex, mean erythrocyte volume and total bilirubin. The AUC of RDW-CV for the predictor of CALs in patients with incomplete KD was 0.597 (95\% confidence interval 
Table 1 Demographic, laboratory characteristics of patients with complete Kawasaki disease

\begin{tabular}{|c|c|c|c|c|}
\hline Variables & All $(n=1060)$ & $\mathrm{CAL}+(n=482)$ & $\mathrm{CAL}-(n=578)$ & $p$ value \\
\hline Age (month) & $29(17-46)$ & $27(17-42.25)$ & $32.00(17.00-48.00)$ & 0.103 \\
\hline Male sex $(\%)$ & $669(63.1 \%)$ & $326(67.6 \%)$ & $343(59.3 \%)$ & 0.005 \\
\hline IVIG resistance (\%) & $41(3.9 \%)$ & $26(5.4 \%)$ & $15(2.6 \%)$ & 0.019 \\
\hline White blood cell (109/L) & $14.18(10.88-17.78)$ & $14.47(11.1-18.29)$ & $13.93(10.82-17.50)$ & 0.144 \\
\hline Platelet count (109/L) & $385(311-484.75)$ & $382.5(311.0-492.25)$ & $387(310.5-478)$ & 0.879 \\
\hline Hemoglobin (g/l) & $108(101-114)$ & $106(99.0-113.0)$ & $110(104-115)$ & 0.000 \\
\hline Mean erythrocyte volume (fL) & $79.9(77.7-82.2)$ & 79.75 (77.6-81.9) & $80.0(77.9-82.4)$ & 0.085 \\
\hline RDW-SD (fL) & $39(37-41)$ & $39(38-41)$ & $38(37-40)$ & 0.000 \\
\hline RDW-CV (\%) & $13.3(12.8-14)$ & $13.5(13.0-14.23)$ & $13.2(12.7-13.72)$ & 0.000 \\
\hline Percentage of neutrophil & $0.73(0.62-0.82)$ & $0.73(0.62-0.82)$ & $0.73(0.63-0.82)$ & 0.600 \\
\hline Percentage of lymphocyte & $0.22(0.14-0.31)$ & $0.22(0.14-0.32)$ & $0.22(0.14-0.31)$ & 0.827 \\
\hline Lymphocyte/neutrophil & $0.30(0.17-0.50)$ & $0.31(0.17-0.51)$ & $0.30(0.17-0.49)$ & 0.821 \\
\hline Percentage of eosinophils & $0.02(0.01-0.04)$ & $0.02(0.01-0.04)$ & $0.02(0.01-0.04)$ & 0.203 \\
\hline Erythrocyte sedimentation rate $(\mathrm{mm} / \mathrm{L})$ & $73.0(54.0-90.0)$ & $73.0(54.0-90.0)$ & $73.0(55.0-89.0)$ & 0.682 \\
\hline $\mathrm{CRP} \geq 10(\%)$ & $987(93.1 \%)$ & $451(93.6 \%)$ & $536(92.7 \%)$ & 0.628 \\
\hline Procalcitonin & $0.547(0.190-1.72)$ & $0.72(0.22-1.98)$ & $0.46(0.17-1.50)$ & 0.003 \\
\hline Glutamyltranspeptidase (U/L) & $33.0(13.88-111.00)$ & $43.0(15.7-116.0)$ & $27.0(13.0-104.0)$ & 0.005 \\
\hline Alanine transaminase (IU/L) & $31.0(16.9-77.9)$ & $33.1(17.0-74.2)$ & $30.15(16.83-84.68)$ & 0.543 \\
\hline Aspartate aminotransferase (IU/L) & $28.2(22.3-41.1)$ & $27.4(22.0-40.0)$ & $29.0(22.63-44.50)$ & 0.070 \\
\hline AST/ALT & $1.00(0.53-1.64)$ & $0.92(0.51-1.54)$ & $1.07(0.54-1.72)$ & 0.043 \\
\hline Total bile acid (umol/L) & $7.8(4.5-16.5)$ & $8.2(4.7-17.2)$ & $7.2(4.2-14.9)$ & 0.154 \\
\hline Total bilirubin (umol/L) & $5.30(3.30-8.70)$ & $5.30(3.30-8.40)$ & $5.30(3.40-8.80)$ & 0.586 \\
\hline Albumin (g/L) & $37.8(34.5-40.7)$ & $37.0(33.5-39.8)$ & $38.4(35.60-41.20)$ & 0.000 \\
\hline Uric acid (umol) & $194(154.18-244)$ & $191.0(150.0-241.75)$ & $195.2(155.6-247.0)$ & 0.244 \\
\hline Creatinine (umol/L) & $26(22-31)$ & $26.0(22.0-32.0)$ & $26.0(22.0-30.0)$ & 0.114 \\
\hline Serum sodium $(\mathrm{mmol} / \mathrm{L})$ & 136.9 (135.1-138.8) & $136.7(134.9-138.4)$ & $137.1(135.2-139.0)$ & 0.007 \\
\hline Fibrinogen $(\mathrm{g} / \mathrm{L})$ & $6.25(5.00-7.17)$ & $6.24(4.95-7.04)$ & $6.27(5.11-7.37)$ & 0.122 \\
\hline Activated partial thromboplastin time (s) & $30.1(28.0-32.8)$ & $30.3(27.8-32.9)$ & $29.95(28.2-32.78)$ & 0.624 \\
\hline
\end{tabular}

$C A L s$ coronary artery lesions, $C I$ confidence interval, $I V I G$ intravenous immunoglobulin, $R D W$ - $C V$ Red blood cell distribution width variation coefficient, $R D W-S D$ Red blood cell distribution width standard deviation, $C R P$ C-reactive protein, AST Aspartate aminotransferase; ALT Alanine transaminase

$0.532-0.661 ; p=0.004)$, with a sensitivity and a specificity value of $40 \%$ and $77 \%$, respectively, when the optimal cutoff value of RDW-CV was 13.85. To our knowledge, this is the first study to evaluate the association between RDW-SD and CALs in patients with KD.

RDW is routinely measured in clinical practice as part of the CBC. RDW is often expressed as RDW-CV and RDWSD; RDW-SD has better sensitivity and is less affected by other factors compared with RDW-CV [19]. However, studies conducted so far only consider the role of RDW$\mathrm{CV}$ as a predictor of CALs in patients with KD. Xu et al. [20] based their study on 422 patients with KD who were followed up for a period of 3 months. They observed that a baseline RDW-CV above $14.55 \%$ is an independent predictor of CALs (odds ratio 3.422, 95\% confidence interval 2.014-6.045, $p=0.000)$. Similarly, Zhu et al. [21] revealed that an elevated RDW-CV value of $>13.35 \%$ is associated with CALs in patients with KD. Unfortunately, in our study, RDW-CV is only an independent risk factor for CALs in patients with incomplete KD. In patients with complete KD, although univariate analyses showed that RDW-CV is a significant predictor of CALs, significance was not retained with multivariate logistic regression. Compared with studies by $\mathrm{Xu}$ et al. and $\mathrm{Zhu}$ et al., we controlled for additional confounding factors, including age, sex and hemoglobin. In our study, RDW-SD is an independent predictor of CALs in patients with complete KD. RDW-SD may therefore outperform RDW-CV as a predictor of CALs in patients with complete KD.

RDW values are typically elevated under conditions of ineffective RBC production, increased RBC destruction, and blood transfusion. Nevertheless, the pathophysiological path of increased RDW in patients with KD with CALs remains unknown. Although the precise mechanism by 
Table 2 Demographic, laboratory characteristics of patients with incomplete Kawasaki disease

\begin{tabular}{|c|c|c|c|c|}
\hline Variables & All $(n=295)$ & $\mathrm{CAL}+(n=154)$ & $\mathrm{CAL}-(n=141)$ & $p$ value \\
\hline Age (month) & $28(15-53)$ & $26(15.75-47.25)$ & $34(14.5-57.0)$ & 0.253 \\
\hline Male sex $(\%)$ & $187(63.4 \%)$ & $107(69.5 \%)$ & $80(56.7 \%)$ & 0.023 \\
\hline IVIG resistance (\%) & $4(1.4 \%)$ & $2(1.4 \%)$ & $2(1.3 \%)$ & 0.929 \\
\hline White blood cell (109/L) & $12.29(8.88-15.88)$ & $12.2(8.29-17.22)$ & $12.35(9.31-14.88)$ & 0.651 \\
\hline Platelet count (109/L) & $406(309-540)$ & $407(285.25-568.75)$ & $406(324.5-513)$ & 0.803 \\
\hline Hemoglobin (g/l) & $108(101-117)$ & $108(100.75-116.25)$ & $110(102-118)$ & 0.273 \\
\hline Mean erythrocyte volume (fL) & $79.9(77.6-82.3)$ & $79.5(76.5-82.4)$ & $80.5(78.4-82.15)$ & 0.022 \\
\hline RDW-SD (fL) & $39(37-41)$ & $39(37-41)$ & $39(37-41)$ & 0.219 \\
\hline RDW-CV (\%) & $13.3(12.8-14.2)$ & $13.55(12.9-14.3)$ & $13.3(12.7-13.8)$ & 0.004 \\
\hline Percentage of neutrophil & $0.63(0.52-0.72)$ & $0.61(0.48-0.70)$ & $0.65(0.55-0.74)$ & 0.150 \\
\hline Percentage of lymphocyte & $0.30(0.22-0.41)$ & $0.32(0.24-0.43)$ & $0.29(0.21-0.40)$ & 0.069 \\
\hline Lymphocyte/neutrophil & $0.47(0.31-0.79)$ & $0.52(0.35-0.92)$ & $0.44(0.28-0.73)$ & 0.112 \\
\hline Percentage of eosinophils & $0.02(0.01-0.04)$ & $0.02(0.01-0.04)$ & $0.02(0.01-0.04)$ & 0.474 \\
\hline Erythrocyte sedimentation rate $(\mathrm{mm} / \mathrm{L})$ & $66.5(48.0-85.75)$ & $65.5(45.25-83.75)$ & $69.5(51.0-88.0)$ & 0.230 \\
\hline $\mathrm{CRP} \geq 10(\%)$ & $239(81 \%)$ & $119(77.3 \%)$ & $120(85.1 \%)$ & 0.102 \\
\hline Procalcitonin & $0.27(0.11-0.71)$ & $0.28(0.10-0.66)$ & $0.27(0.11-0.79)$ & 0.612 \\
\hline Glutamyltranspeptidase (U/L) & $16.0(11.0-39.75)$ & $17.0(11.6-40.0)$ & $13.1(10.1-39.0)$ & 0.100 \\
\hline Alanine transaminase (IU/L) & $21.6(13.2-38.12)$ & $23.7(14.0-38.9)$ & $19.8(12.65-36.85)$ & 0.406 \\
\hline Aspartate aminotransferase (IU/L) & $29.00(22.65-40.95)$ & $29.9(22.8-41.4)$ & $26.7(22.0-40.6)$ & 0.269 \\
\hline AST/ALT & $1.45(0.97-2.13)$ & $1.45(0.94-2.08)$ & $1.45(1.01-2.15)$ & 0.750 \\
\hline Total bile acid (umol/L) & $5.70(3.20-10.93)$ & $5.30(3.20-10.30)$ & $5.70(3.20-11.8)$ & 0.361 \\
\hline Total bilirubin (umol/L) & $3.85(2.50-6.00)$ & $3.40(2.30-5.20)$ & $4.3(2.9-6.95)$ & 0.007 \\
\hline Albumin (g/L) & $39.90(37.10-42.70)$ & $39.4(35.8-42.2)$ & $40.2(37.65-43.00)$ & 0.053 \\
\hline Uric acid (umol) & $195.8(150.5-244.3)$ & $202.0(154.0-254.0)$ & $189.0(147.0-236.2)$ & 0.250 \\
\hline Creatinine (umol/L) & $26.00(22.00-31.95)$ & $25.1(22.0-31.0)$ & $26.0(22.15-32.0)$ & 0.406 \\
\hline Serum sodium $(\mathrm{mmol} / \mathrm{L})$ & $138.3(136.6-139.8)$ & $138.1(136.28-139.9)$ & $138.3(136.8-139.6)$ & 0.856 \\
\hline Fibrinogen $(\mathrm{g} / \mathrm{L})$ & $5.57(3.95-6.86)$ & $5.13(3.82-6.84)$ & $5.89(4.36-6.94)$ & 0.156 \\
\hline Activated partial thromboplastin time (s) & $29.6(27.2-32.15)$ & $29.5(27.25-31.75)$ & $29.6(27.0-32.55)$ & 0.615 \\
\hline
\end{tabular}

$C A L s$ coronary artery lesions, $C I$ confidence interval, $I V I G$ intravenous immunoglobulin, $R D W$ - $C V$ Red blood cell distribution width variation coefficient, $R D W$-SD Red blood cell distribution width standard deviation, $C R P$ C-reactive protein, AST Aspartate aminotransferase, $A L T$ Alanine transaminase

which elevated RDW is associated with CALs in patients with $\mathrm{KD}$ is beyond the scope of this study, several explanations are possible. One of the possible mechanisms is inflammation, which plays an important role in the process of $\mathrm{KD}$. In the acute phase of KD, the levels of various inflammatory cytokines, such as tumor necrosis factor- $\alpha$, interleukin (IL)-1, IL-2, and IL-6, are relatively high and contribute to CAL formation [22-25]. Demir et al. [26] found that patients with KD and CALs have more severe inflammation compared with patients without CALs; thus, markers such as neutrophil-lymphocyte ratio, neutrophil count, and C-reactive protein, differed significantly in this population. Inflammatory states are strongly related to ineffective erythropoiesis, and the release of inflammatory cytokines may increase anisocytosis by suppressing erythropoietin activity and inhibiting erythrocyte maturation [27]. Neurohormonal activation may also contribute to an increase in RDW in patients with KD and CALs. N-terminal pro-brain natriuretic peptide (NT-proBNP), which is an important index of the neurohormonal pathway, is a valuable biomarker for predicting CALs in patients with KD [28, 29]. In our study, we did not measure NT-proBNP; however, Fukuta et al. [30] found that even after adjusting for numerous potential confounders, elevated BNP levels were independently associated with a higher RDW in patients with coronary artery disease.

Additionally, Kim et al. [31] demonstrated that indicators of iron deficiency anemia, especially ferritin, were highly associated with coronary artery abnormalities. Kuo et al. [32] showed that inflammation-induced hepcidin is associated with anemia development and outcomes in patients with KD. High levels of hepcidin lead to a low serum iron content and limit availability of iron for erythropoiesis, resulting in an increase in RDW [33]. However, no indices reflecting iron metabolism were available in our study; therefore, we 
Table 3 Logistic regression analysis of Kawasaki disease with CALs

\begin{tabular}{lllll}
\hline KD type & Variables & OR & $95 \%$ CI & $p$ value \\
\hline Complete Kawasaki disease & Age & 1.006 & $1.000-1.012$ & 0.050 \\
& Male sex & 0.669 & $0.503-0.888$ & 0.006 \\
& IVIG resistance & 1.661 & $0.795-3.469$ & 0.177 \\
& Hemoglobin & 0.974 & $0.958-0.991$ & 0.003 \\
& Glutamyltranspeptidase & 1.000 & $0.999-1.001$ & 0.965 \\
& Procalcitonin & 1.007 & $0.981-1.034$ & 0.609 \\
& Albumin & 0.971 & $0.938-1.001$ & 0.099 \\
& Serum sodium & 0.960 & $0.916-1.007$ & 0.093 \\
& AST/ALT & 0.975 & $0.885-1.075$ & 0.610 \\
& RDW-CV & 1.078 & $0.894-1.301$ & 0.432 \\
& RDW-SD & 1.115 & $1.046-1.188$ & 0.001 \\
& Age & 1.003 & $0.994-1.013$ & 0.464 \\
& Male sex & 0.634 & $0.385-1.046$ & 0.074 \\
& Mean erythrocyte volume & 0.977 & $0.917-1.042$ & 0.485 \\
& Total bilirubin & 0.931 & 0.861 & 1.007 \\
& RDW-CV & 1.428 & $1.056-1.930$ & 0.021 \\
\hline
\end{tabular}

$C A L s$ coronary artery lesions, $C I$ confidence interval, IVIG intravenous immunoglobulin, $O R$ odds ratio, $R D W$ - $C V$ Red blood cell distribution width variation coefficient, $R D W$-SD Red blood cell distribution width standard deviation

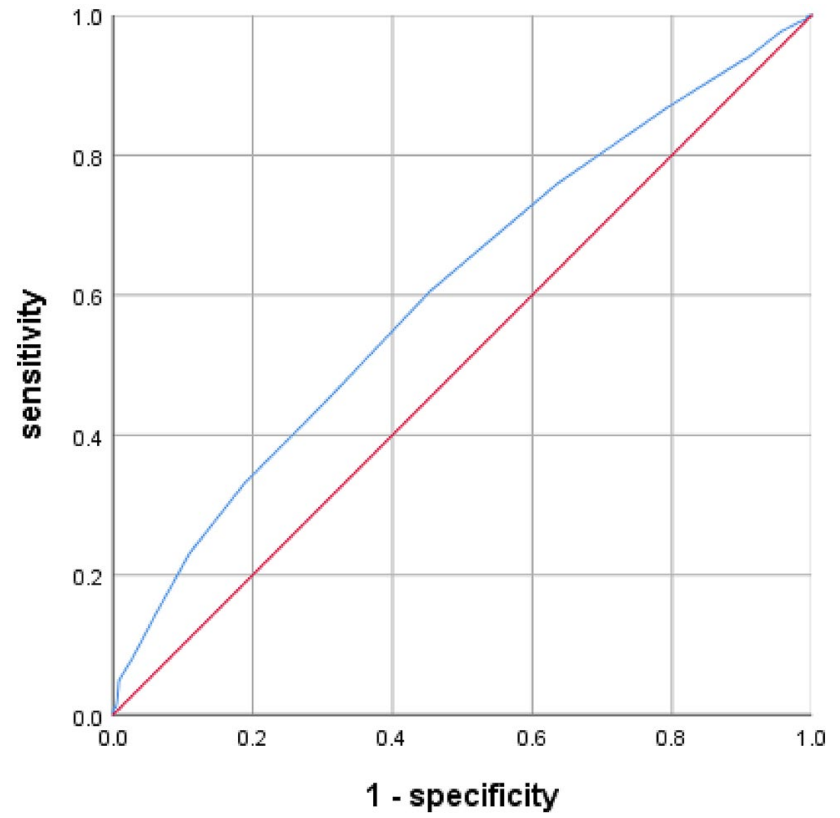

Fig. 1 Receiving Operating Characteristic (ROC) of Red blood cell distribution width standard deviation (RDW-SD) for predicting coronary artery lesions in patients with complete Kawasaki disease

were unable to confirm a relationship between RDW and iron metabolism. We found that patients with KD and CALs had a lower mean erythrocyte volume, which may indirectly reflect iron deficiency. Thus, impaired iron mobilization may be a pathophysiological mechanism of the association between increased RDW and CALs.

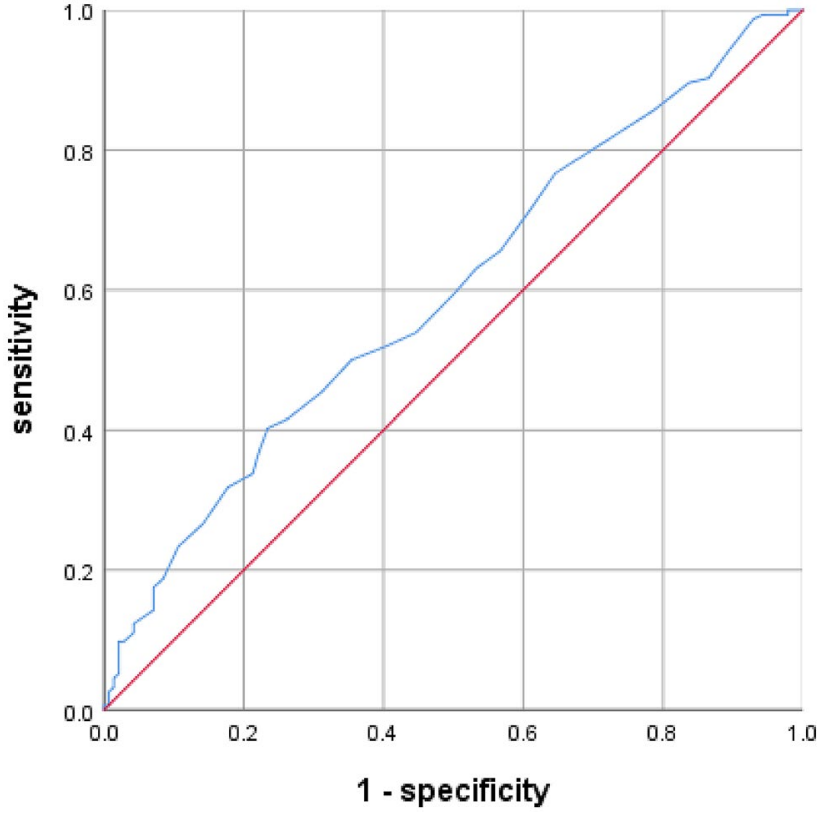

Fig. 2 Receiving Operating Characteristic (ROC) of Red blood cell distribution width variation coefficient (RDW-CV) for predicting coronary artery lesions in patients with incomplete Kawasaki disease

Nonetheless, several limitations hindered our study. First, this was a retrospective study; therefore, selection bias may exist. Second, due to lack of height data, CALs were assessed according to absolute coronary artery diameter rather than mean BSA-adjusted Z-score. In addition, due to the limited sample size, we did not conduct a subgroup 
analysis of CAL severity. Third, despite adjusting for multiple variables, it is possible that other residual confounding variables influenced the results, such as iron, folate, and vitamin B12. Finally, although an association between an increase in RDW and presence of CALs in patients with KD was established in the present study, whether RDW has a causal role in CAL development or whether it acts as a marker of the disease process needs further investigation.

\section{Conclusion}

In conclusion, RDW-SD is an independent predictor of CALs in patients with complete $\mathrm{KD}$, but this relationship is not observed for incomplete KD. On the contrary, the $\mathrm{RDW}-\mathrm{CV}$ is an independent predictor of CALs in patients with incomplete $\mathrm{KD}$, but not in complete KD. Therefore, we propose that future studies on the correlation between RDW and KD should pay attention to the differences in the types of KD and attach importance to the value of RDW-SD, which does not include MCV in the calculation. Our findings call for further studies to achieve a better understanding of the mechanism by which RDW relates to KD.

Author Contributions LM and GY conceived and designed the study. LM and QL collected the data. LM and HC analyzed the data. LM wrote the first manuscript draft. GY revised it critically for important intellectual content. All authors provided intellectual input, had access to the complete dataset, contributed to manuscript revisions and approved of the final version. GY is the guarantor.

Funding This work was supported by the Key Project of Medical Research Program of Chongqing Health Bureau (2012-1-047).

Data Availability The data and material are available from the corresponding author on reasonable request.

\section{Declarations}

Conflict of interest All authors declare no competing interests.

Ethical Approval This study was discussed by the Ethics Committee of the Children's Hospital Affiliated to Chongqing Medical University, no objection was made (2020.223).

Consent for Publication All authors consent for publication.

Open Access This article is licensed under a Creative Commons Attribution 4.0 International License, which permits use, sharing, adaptation, distribution and reproduction in any medium or format, as long as you give appropriate credit to the original author(s) and the source, provide a link to the Creative Commons licence, and indicate if changes were made. The images or other third party material in this article are included in the article's Creative Commons licence, unless indicated otherwise in a credit line to the material. If material is not included in the article's Creative Commons licence and your intended use is not permitted by statutory regulation or exceeds the permitted use, you will need to obtain permission directly from the copyright holder. To view a copy of this licence, visit http://creativecommons.org/licenses/by/4.0/.

\section{References}

1. Kawasaki T (1967) Acute febrile mucocutaneous syndrome with lymphoid involvement with specific desquamation of the fingers and toes in children. Arerugi 16(3):178-222

2. Lo MS, Newburger JW (2018) Role of intravenous immunoglobulin in the treatment of Kawasaki disease. Int J Rheum Dis 21(1):64-69

3. Mori M, Imagawa T, Yasui K, Kanaya A, Yokota S (2000) Predictors of coronary artery lesions after intravenous gamma-globulin treatment in Kawasaki disease. J Pediatr 137(2):177-180

4. Brogan P, Burns JC, Cornish J, Diwakar V, Eleftheriou D, Gordon JB, Gray HH, Johnson TW, Levin M, Malik I, MacCarthy P, McCormack R, Miller O, Tulloh R (2020) Lifetime cardiovascular management of patients with previous Kawasaki disease. Heart 106(6):411-420

5. McCrindle BW, Rowley AH, Newburger JW, Burns JC, Bolger AF, Gewitz M, Baker AL, Jackson MA, Takahashi M, Shah PB, Kobayashi T, Wu MH, Saji TT, Pahl E (2017) Diagnosis, treatment, and long-term management of Kawasaki disease: a scientific statement for health professionals from the American Heart Association. Circulation 135(17):e927-e999

6. Burns JC, Glodé MP (2004) Kawasaki syndrome. Lancet 364(9433):533-544

7. Mawlana W, Donia A, Elamrousy D (2014) Relation between red cell distribution width and left ventricular function in children with heart failure. ISRN Pediatr. https://doi.org/10.1155/2014/ 234835

8. Felker GM, Allen LA, Pocock SJ, Shaw LK, McMurray JJ, Pfeffer MA, Swedberg K, Wang D, Yusuf S, Michelson EL, Granger CB (2007) Red cell distribution width as a novel prognostic marker in heart failure: data from the CHARM Program and the Duke Databank. J Am Coll Cardiol 50(1):40-47

9. Li Y, Li M, Teng Y, Zhang C, Liu Q, Hou H (2018) The association between red cell distribution width, erythropoietin levels, and coronary artery disease. Coron Artery Dis 29(1):74-80

10. Buyukkaya E, Erayman A, Karakas E, Bugra NA, Kurt M, Buyukkaya S, Burak AA, Sen N (2016) Relation of red cell distribution width with dipper and non-dipper hypertension. Med Glas (Zenica) 13(2):75-81

11. Liu S, Wang P, Shen PP, Zhou JH (2016) Predictive values of red blood cell distribution width in assessing severity of chronic heart failure. Med Sci Monit 22:2119-2125

12. Demirtas S, Karahan O, Yazici S, Guclu O, Caliskan A, Yavuz C, Kucuker A, Mavitas B (2014) The relationship between complete blood count parameters and Fontaine's stages in patients with peripheral arterial disease. Vascular 22(6):427-431

13. Li XL, Hong LF, Jia YJ, Nie SP, Guo YL, Xu RX, Zhu CG, Jiang LX, Li JJ (2014) Significance of red cell distribution width measurement for the patients with isolated coronary artery ectasia. $\mathrm{J}$ Transl Med 12:62

14. Baggen V, van den Bosch AE, van Kimmenade RR, Eindhoven JA, Witsenburg M, Cuypers J, Leebeek F, Boersma E, Roos-Hesselink JW (2018) Red cell distribution width in adults with congenital heart disease: a worldwide available and low-cost predictor of cardiovascular events. Int J Cardiol 260:60-65

15. Fava C, Cattazzo F, Hu ZD, Lippi G, Montagnana M (2019) The role of red blood cell distribution width (RDW) in cardiovascular risk assessment: useful or hype? Ann Transl Med 7(20):581 
16. Hoffmann JJ, Nabbe KC, van den Broek NM (2015) Effect of age and gender on reference intervals of red blood cell distribution width (RDW) and mean red cell volume (MCV). Clin Chem Lab Med 53(12):2015-2019

17. Tonelli M, Wiebe N, James MT, Naugler C, Manns BJ, Klarenbach SW, Hemmelgarn BR (2019) Red cell distribution width associations with clinical outcomes: a population-based cohort study. PLoS ONE 14(3):e212374

18. Qiu H, Jia C, Wang Z, He Y, Rong X, Wu R, Chu M, Shi H (2020) Prognosis and risk factors of coronary artery lesions before immunoglobulin therapy in children with Kawasaki disease. Balkan Med J 37(6):324-329

19. Zhang FX, Li ZL, Zhang ZD, Ma XC (2019) Prognostic value of red blood cell distribution width for severe acute pancreatitis. World J Gastroenterol 25(32):4739-4748

20. Xu H, Fu S, Wang W, Zhang Q, Hu J, Gao L, Zhu W, Gong F (2016) Predictive value of red blood cell distribution width for coronary artery lesions in patients with Kawasaki disease. Cardiol Young 26(6):1151-1157

21. Zaifu Z, Xiaochen F (2020) Changes and significance of red blood cell distribution width of Kawasaki disease in children. J Clin Pediatr 38(3): 182-185

22. Hui-Yuen JS, Duong TT, Yeung RS (2006) TNF-alpha is necessary for induction of coronary artery inflammation and aneurysm formation in an animal model of Kawasaki disease. J Immunol 176(10):6294-6301

23. Lee Y, Schulte DJ, Shimada K, Chen S, Crother TR, Chiba N, Fishbein MC, Lehman TJ, Arditi M (2012) Interleukin-1 $\beta$ is crucial for the induction of coronary artery inflammation in a mouse model of Kawasaki disease. Circulation 125(12):1542-1550

24. Si F, Wu Y, Gao F, Feng S, Liu R, Yi Q (2017) Relationship between IL-27 and coronary arterial lesions in children with Kawasaki disease. Clin Exp Med 17(4):451-457

25. Su Y, Feng S, Luo L, Liu R, Yi Q (2019) Association between IL-35 and coronary arterial lesions in children with Kawasaki disease. Clin Exp Med 19(1):87-92

26. Demir F, Karadeniz C, Özdemir R, Yozgat Y, Çelegen K, Karaaslan U, Demirol M, Meşe T, Ünal N (2015) Usefulness of neutrophil to lymphocyte ratio in prediction of coronary artery lesions in patients with Kawasaki disease. Balkan Med J 32(4):371-376

27. Isik T, Kurt M, Ayhan E, Uyarel H, Tanboga IH, Korkmaz AF, Kaya A, Sevimli S (2012) Relation of red cell distribution width with presence and severity of coronary artery ectasia. Clin Appl Thromb Hemost 18(5):441-447

28. Yoshimura K, Kimata T, Mine K, Uchiyama T, Tsuji S, Kaneko K (2013) N-terminal pro-brain natriuretic peptide and risk of coronary artery lesions and resistance to intravenous immunoglobulin in Kawasaki disease. J Pediatr 162(6):1205-1209

29. Jung JY, Ham EM, Kwon H, Kwak YH, Kim DK, Lee JH, Jung JH (2019) N-terminal pro-brain natriuretic peptide and prediction of coronary artery dilatation in hyperacute phase of Kawasaki disease. Am J Emerg Med 37(3):468-471

30. Fukuta H, Ohte N, Mukai S, Saeki T, Asada K, Wakami K, Kimura G (2009) Elevated plasma levels of B-type natriuretic peptide but not $\mathrm{C}$-reactive protein are associated with higher red cell distribution width in patients with coronary artery disease. Int Heart J 50(3):301-312

31. Kim S, Eun LY (2019) Iron deficiency anemia as a predictor of coronary artery abnormalities in Kawasaki disease. Korean J Pediatr 62(8):301-306

32. Kuo HC, Yang YL, Chuang JH, Tiao MM, Yu HR, Huang LT, Yang KD, Chang WC, Lee CP, Huang YH (2012) Inflammationinduced hepcidin is associated with the development of anemia and coronary artery lesions in Kawasaki disease. J Clin Immunol 32(4):746-752

33. Allen LA, Felker GM, Mehra MR, Chiong JR, Dunlap SH, Ghali JK, Lenihan DJ, Oren RM, Wagoner LE, Schwartz TA, Adams KJ (2010) Validation and potential mechanisms of red cell distribution width as a prognostic marker in heart failure. J Cardiac Fail 16(3):230-238

Publisher's Note Springer Nature remains neutral with regard to jurisdictional claims in published maps and institutional affiliations. 